OPEN ACCESS

Edited by:

Maoshan Chen,

Monash University, Australia

Reviewed by:

Nadia Rucci,

University of L'Aquila, Italy

Wanessa Altei,

Federal University of Sao Carlos, Brazil

${ }^{*}$ Correspondence:

Flavia Pichiorri

fpichiorri@coh.org

Specialty section:

This article was submitted to

Molecular and Cellular Oncology,

a section of the journal

Frontiers in Oncology

Received: 01 April 2021

Accepted: 13 May 2021

Published: 21 June 2021

Citation:

Khalife J, Sanchez JF and Pichiorri F (2021) The Emerging

Role of Extracellular VesicleAssociated RNAs in the Multiple

Myeloma Microenvironment.

Front. Oncol. 11:689538. doi: 10.3389/fonc.2021.689538

\section{The Emerging Role of Extracellular Vesicle-Associated RNAs in the Multiple Myeloma Microenvironment}

\author{
Jihane Khalife ${ }^{1,2}$, James F. Sanchez ${ }^{1}$ and Flavia Pichiorri ${ }^{1,2 *}$ \\ 1 Judy and Bernard Briskin Center for Multiple Myeloma Research, City of Hope, Duarte, CA, United States, ${ }^{2}$ Department of \\ Hematologic Malignancies Translational Science, City of Hope, Duarte, CA, United States
}

Multiple myeloma (MM) is a cancer of terminally differentiated plasma cells (PCs) that develop at multiple sites within the bone marrow (BM). MM is treatable but rarely curable because of the frequent emergence of drug resistance and relapse. Increasing evidence indicates that the BM microenvironment plays a major role in supporting MM-PC survival and resistance to therapy. The BM microenvironment is a complex milieu containing hematopoietic cells, stromal cells, endothelial cells, immune cells, osteoclasts and osteoblasts, all contributing to the pathobiology of MM, including PC proliferation, escape from immune surveillance, angiogenesis and bone disease development. Small extracellular vesicles (EVs) are heterogenous lipid structures released by all cell types and mediate local and distal cellular communication. In MM, EVs are key mediators of the cross-talk between PCs and the surrounding microenvironment because of their ability to deliver bioactive cargo molecules such as lipids, mRNAs, non-coding regulatory RNA and proteins. Hence, MM-EVs highly contribute to establish a tumor-supportive BM niche that impacts MM pathogenesis and disease progression. In this review, we will first highlight the effects of RNA-containing, MM-derived EVs on the several cellular compartments within the BM microenvironment that play a role in the different aspects of MM pathology. We will also touch on the prospective use of MM-EV-associated non-coding RNAs as clinical biomarkers in the context of "liquid biopsy" in light of their importance as a promising tool in $\mathrm{MM}$ diagnosis, prognosis and prediction of drug resistance.

Keywords: multiple myeloma, microenvironment, extracellular vesicles, non-coding RNA, biomarker

\section{INTRODUCTION}

Multiple myeloma is a hematological malignancy characterized by clonal expansion of plasma cells (PCs) at different sites within the bone marrow (BM). It progresses from monoclonal gammopathy of undetermined significance (MGUS) and smoldering myeloma (SMM). Diagnosis may include the presence of more than $10 \%$ of malignant plasma cells in the BM. Despite the introduction over the last two decades of novel treatments such as proteasome inhibitors and immunomodulatory drugs that significantly improved patients' survival, MM is still incurable, typically with multiple episodes of remission and relapse (1). One of the reasons why MM is highly heterogenous and variable in survival is that its pathogenesis is strongly associated with a BM-tumor supportive environment. 
MM-PCs highly depend on various components of the BM microenvironment for growth and survival. The dynamic cross-talk between MM-PCs and cells of the BM microenvironment play a crucial role in modifying the oncogenic landscape of the microenvironment in favor of disease occurrence, progression and resistance to treatment $(1,2)$. The BM microenvironment is a complex milieu composed of a cellular and non-cellular compartment. The cellular compartment contains hematopoietic cells, mesenchymal stromal cells, endothelial cells, immune cells, osteoclasts and osteoblasts, all contributing to the pathobiology of MM, including PC proliferation, escape from immune surveillance, angiogenesis and bone disease development (2).

Extracellular vesicles (EVs) are lipid bilayers that are heterogenous in size and content; they are released by all cell types, both normal and malignant, known to be involved in cellcell communication at short and long distances (3). EVs were first recognized as a route of cellular waste release (4). However, more studies done on their biogenesis and content have revealed novel findings that have implication in transfer of information in both health and disease (5). In MM, EVs play a pivotal role in mediating a mutual communication between MM-PCs and cells of the tumor microenvironment by transferring bioactive molecules such as lipids, proteins, and regulatory RNAs, all contributing to MM pathobiology (6). Moreover, EVs are abundantly released in the sera of MM patients carrying selective molecular cargoes indicative of disease state (7). Therefore, MM-EVs play an essential role as biomarkers of disease in the context of liquid biopsies, where solid biopsies have limited accessibility.

The RNA cargo of EVs mainly consists of messenger RNA (mRNA) and non-coding RNA (ncRNA) and varies among different biofluids (8) and among vesicles from different cell types (9). In this context, non-coding RNAs (microRNAs, piwiRNAs, long non-coding RNAs and circular RNAs) were shown to be the most enriched nucleic acid bioactive cargoes in the EVs of cancer patients (10). The biological functions of EV-regulatory RNAs were well identified in solid tumors and were the subject of phase I clinical trials (11-13). However, their role in hematological malignancies is in the process of being defined. In this review, we will focus on discussing the effects of RNAcontaining, MM-derived EVs on the cellular compartments within the $\mathrm{BM}$ microenvironment that play a crucial role in the various aspects of MM pathology. We will also highlight the important role of circulating EV-non-coding RNAs as diagnostic markers and biomarkers of MM progression and drug resistance in the context of liquid biopsy.

\section{Extracellular Vesicles: Biogenesis and Trafficking}

The International Society for Extracellular Vesicles (ISEV) uses EVs as a general description to all particles, released by all cell types, that lack a nucleus but exhibit lipid bilayers. EVs are classified according to different features, including orders of size, mechanism of biogenesis, cell of origin and cargo content. According to the updated guidelines of Minimal Information for Studies of Extracellular Vesicles 2018 (14), assigning EVs on the basis of their biogenesis pathways or subtype markers remains difficult. Therefore, MISEV18 recommends to name EVs in accordance with their physical characteristics such as size, biochemical composition, or described conditions. Herein, the main classification of EVs will be their order of size, aligned with the latest classification of MISEV2018. Two main subgroups have been defined: i) small EVs; ii) medium/large EVs. Both subgroups contain several subclasses based on cellular origin, morphology, function and biogenesis $(14,15)$.

i. Small EVs (sEVs) represent the smallest EVs. They are less than $100 \mathrm{~nm}$ or $200 \mathrm{~nm}$ in size. Exosomes are an example of sEVs. They range in size between 30 to $150 \mathrm{~nm}$. They are derived from the endocytic compartment and undergo further maturation to form intraluminal vesicles. They carry a wide variety of cargo molecules including RNAs (mRNAs and non-coding regulatory RNAs) and proteins. EVencapsulated mRNAs can be translated to proteins by the recipient cells $(14,16)$. Retrovirus-like particles are another example of small EVs. They range between $90-100 \mathrm{~nm}$ in size, they directly bud from the plasma membrane, and they contain retroviral particles, cytoskeleton proteins, or plasma membrane components (17).

ii. Medium/large EVs (m/lEVs) $>200 \mathrm{~nm}$ in size (14). Some types of microvesicles or ectosomes that range in size from 200 to $1000 \mathrm{~nm}$ can be considered medium EVs. They are formed by plasma membrane budding. They release their cargoes by remodeling the cortical cytoskeleton. Unlike exosomes, they are not formed in a consistent manner but by specific cell types. Their cargoes consist of RNAs and proteins similar to exosomes. However, microvesicles are the only particles that can additionally encapsulate plasmid DNA $(18,19)$. Large vesicles reach up to $10,000 \mathrm{~nm}$ in size and contain cytoskeletal structures $(14,20,21)$. Apoptotic bodies are an example of large vesicles with a diameter greater than $1000 \mathrm{~nm}$. Their name is based on the description of condition of cell of origin (14). They are formed from cleavage of the cytoplasmic membrane upon activation of apoptosis or programmed cell death. They contain both cytosolic components (RNA and protein) and nuclear fragments (22).

EVs are released by all cell types under both physiological conditions and stress responses. They carry specific bioactive molecules (RNA, protein), and their composition is strictly dependent on the parental cells from which they originated. They release their content in the surrounding interstitial space or biofluids (urine, blood), where they are named "circulating vesicles," or they can be taken up by recipient cells at short or long distances, facilitating cell-cell communication (23). At the destination site, EVs release their cargoes by several mechanisms, including membrane fusion, phagocytosis, receptor-mediated caveolin-clathrin or endocytosis mediated by lipid rafts, enabling specific molecular functions in the recipient cells (24). In recent years, the close association between EVs and tumor cell survival has been a major focus of research. Indeed, tumor cells have been shown to secrete dramatically higher quantities of EVs 
in the plasma than their healthy counterparts. The majority of EVs secreted by malignant cells contained specific cargo molecules involved in tumor development, further supporting the role of EVs in tumor growth and disease progression (25).

\section{CHARACTERIZATION OF MM-PCs AND MM-BM CELLS-DERIVED EVs}

Malignant PCs often home at multiple sites within the BM. Because MM extensively relies on the microenvironment for development, EVs are considered essential mediators by which MM cells disrupt BM microenvironment homeostasis in favor of tumor development (26). Although to date, standardized methods for proper isolation and characterization of ultrapure EVs are still lacking (27), a high-throughput technique has been purposely applied to characterize MM-derived EVs for optimal functional analysis of their contents using a minimum amount of starting material (28).

We have used global systemic proteomic analysis to characterize EVs isolated from MM and have identified bioactive proteins involved in multiple biological processes that contribute to MM development. For instance, we have identified elevated levels of CD44, MHC class I and bone marrow stromal antigen 2 (BST-2) in MM-derived EVs, with respect to their healthy counterparts. Moreover, levels of membrane molecules such as CD38, CD44, and CD9 were significantly higher in Ig light chain-positive EVs from MM patients as compared to those isolated from MGUS (29). At the RNA level, Manier et al. reported that small noncoding microRNAs were the most predominant regulatory RNAs differentially expressed in MM patients and cancer-free controls. For instance, a small RNA sequencing of circulating EVs identified 158 differentially expressed exosomal microRNAs (30), among which some were previously identified as oncogenic targets in malignant PCs (31) and some were significantly differentially expressed between high and low-risk MM (32). Importantly, exosomal microRNAs were not only released by malignant PCs but also by cells of the microenvironment to exert a mutual communication between MM cells and the BM microenvironment. Indeed, microarray studies revealed that MM-bone marrow stromal cells (MMBMSCs) initiated the transfer of EVs carrying functional microRNAs, resulting in MM cell proliferation, survival and migration, whereas EVs isolated from BMSCs of healthy controls contained a different microRNAs profile and instead inhibited MM tumor growth $(33,34)$. Although studies have specifically demonstrated the involvement of exosomal microRNAs in a crosstalk between MM-PCs and cells of the BM microenvironment (30, 33,35 ), an analysis of EV-associated regulatory RNAs on a wider spectrum is yet to be defined. More studies on a larger number of PCs isolated from MM patients using deep exosomal RNAsequencing are needed to further unravel the contribution of EV-RNAs other than microRNAs in MM pathogenesis. We will review what has been so far demonstrated about the role of EVassociated regulatory RNAs in the cross-talk between MM-PCs and compartments of the BM microenvironment that influence
MM pathogenesis, including tumor growth, immune evasion, angiogenesis and bone disease development.

\section{MM-EV-RNAS AND MESENCHYMAL STEM CELLS (MSCs)}

Bone marrow stromal cells consist of diverse cell populations including BM mesenchymal stem cells (MSCs), fibroblasts, osteoblasts, chondrocytes and adipocytes. They play an essential role in supporting hematopoietic cell development and maturation. In cancer, stromal cells acquire a distinct molecular signature that allows them to evolve in concordance with the disease (36). In MM, BMSCs play a crucial role in disease development and progression by either direct cell-cell contact or indirectly through a mutual communication via EVs. Such interactions result in the release of pro-tumorigenic factors by BMSCs that contribute to MM pathobiology (37). The content of BM-MSC-derived exosomes from MM patients was analyzed and revealed a major breakthrough in the field of vesicular RNA biology. MM-BM-MSC-EVs carrying low levels of the tumor suppressor $m i R-15 a$ were found to be the cause of deregulation of several signaling pathways, including the Akt pathway. Thus, MM-MSCs-derived EVs were engulfed by MM-PCs and induced the proliferation, survival, and migration of the latter by activating oncogenic factors known to be targets of the Akt pathway $(33,38)$. These findings highlight the importance of EVRNAs in supporting MM pathogenesis. Interestingly, a recent study showed that a long non-coding RNA LINCOO461 that acts as a decoy for miR-15a and miR-16 tumor suppressors was transferred to MM cells via MSC-derived EVs, resulting in inhibition of both miRNAs and upregulation of their target anti-apoptotic gene BCL2. As a result, EVs enriched in LINCOO461 inhibited apoptosis of MM cells and induced their proliferation (39).

In line with these findings, another study demonstrated that EVs released by BMSCs of MM patients impacted disease survival and progression. In fact, EVs isolated from BMSCs of MM patients contained a distinct microRNA profile from those isolated from healthy controls. In particular, oncomiRNA $m i R$ $10 a$ was transferred in EVs at high levels to MM cells, resulting in the proliferation of MM-PCs. In MM cells, $m i R-10 a$ was shown to positively regulate the expression of $\beta$-TRC (a key regulator of I $\kappa \mathrm{B}-\alpha$ degradation in the NF- $\kappa \mathrm{B}$ pathway). Inhibiting $\beta$-TRC abrogated miR-10a-mediated MM cell proliferation (34). However, a study showed that MSCs isolated from healthy individuals acquired the tumor phenotype and molecular signature of MSCs from MM patients only when cocultured with malignant PCs (40). Thus, whether MM cells first educate stromal cells and modify their homeostasis in favor of PC survival is not well understood. More work has to be done in this regard to clarify whether MM-PCs are the first initiators of a cross-talk via EVs. For instance, several studies demonstrated that MM-PCs release high amounts of EVs in circulation as compared to normal PCs. Those EVs have been shown to associate with $\mathrm{MM}$ patients' survival and can serve as useful 
biomarkers for prediction of disease progression (24). In the context of the BM microenvironment, EVs from MM-PCs were able to promote proliferation of MSCs and their transformation to cancer-associated fibroblasts (CAFs). CAFs are one of the most dominant stromal cells that play a pivotal role in the MM$\mathrm{BM}$ niche. When transformed, CAFs selectively release protumorigenic soluble factors including tolerogenic cytokines, chemokines that support MM-PCs. In a recent study, Cheng et al. showed that MM cells release EVs carrying high amounts of the oncomiRNA $m i R-21$ and were selectively taken up by BMMSCs. In BM-MSCs, miR-21 activated expression and secretion of the growth factors SDF-1, FAP and $\alpha$-SMA that in turn induced MSC transformation to CAF. As a result, CAFs caused an increase in $\mathrm{MM}$ cell proliferation. Moreover, inhibition of miR-21 in EVs led to a decrease in CAF markers and MM-PC growth (41). In agreement with these findings, another group has observed that MM-EVs were enriched in another oncomiRNA, $m i R-146 a$, known to play a major role in several types of cancer including MM. MM-EVs with high amounts of miR-146a were transferred to MSCs, inducing in the recipient cells an increase in IL-6 secretion, a cytokine known to promote MM cell survival. EV-miR-146a also stimulated the expression and release of growth factors known to increase MM-PCs' viability and migration such as CXCL-1, IP-10, CCL2 and CCL5. This phenomenon was dependent on the $\mathrm{NOTCH}$ signaling pathway. In fact, inhibition of NOTCH signaling in MSCs abrogated the $m i R-146 a$-induced increase in cytokines/ chemokines, as well as MM cell growth and migration (42).

Under normal physiological conditions, MSCs differentiate to osteoblasts known as active bone forming cells. However, MSCs exposed to MM-derived EVs have a reduced capacity to differentiate towards bone nodules, which results in a reduction of bone formation. One mechanism of action that induces such a phenomenon was recently demonstrated by $\mathrm{Li}$ et al. The research group found that bioactive lncRNA RUNX2AS1 in MM cells were encapsulated into EVs and transferred to MSCs. lncRNA RUNX2-AS1 is a long non-coding RNA that represses RUNX2, a key transcription factor that is involved in osteogenic differentiation, by disrupting its splicing. Transfer of lncRNA RUNX2-AS1 resulted in decreased differentiation of MSCs, whereas attenuation of lncRUNX2-AS1 in EV-producing MM cells significantly restored the osteogenic potential of MSCs (43).

\section{MM-EV-RNAs AND OSTEOCLASTS/ OSTEOBLASTS}

MM symptoms include anemia, renal failure and proteinuria. Additionally, one of the major complications associated with MM progression, affecting more than $80 \%$ of patients, is osteolytic bone disease. Patients suffer from persistent bone lesions, leading to pain, fractures, mobility issues and neurological defects (44). Bone formation or osteogenesis involves the collaboration of different group of cells and proceeds through several steps. It requires a tight balance between two major cellular types: osteoblasts and osteoclasts. While osteoblasts, originally differentiated from MSCs in the first step of osteogenesis, are actively involved in bone development; osteoclasts, originating from the fusion of multiple precursor cells, are involved in resorption of bones and reshaping (45). In MM, the balance of this tightly coupled process is wholly impaired, resulting in increased osteoclastic activity together with suppression of osteoblastic activity, both leading to progressive disruption of the bone tissue and osteolytic bone disease (40). The pathological processes and molecular mechanisms involved in MM-related bone disease are well defined. Communication between MM-PCs invading the BM and resident bone cells is believed to be the main cause of shifting the balance to reduced osteoblastogenesis, increased osteoclastogenesis and, ultimately, osteolytic lesions. In fact, once MM cells engraft into the BM, they establish a vicious cycle with bone cells. They have been shown to interact with osteoclasts by expressing essential signaling factors such as MIP-1 $\alpha$, RANKL, MMP-13 and Decoy receptor 3 (DcR3), all known to be responsible for increased osteoclast formation (46-48). Concomitantly, MM cells inhibit osteoblast formation by secreting high amounts of Dickkopf-related protein 1 (DKK1), a key soluble factor known to inhibit the Wnt pathway in osteoblast precursors (pre-osteoblasts). Wnt signaling is essential to promote bone morphogenic protein (BMP-2)-mediated differentiation of pre-osteoblasts to mature osteoblasts in the marrow. In addition to increased osteoclastogenesis, DKK1-induced inhibition of Wnt signaling is an underlying mechanism involved in bone loss in MM (49). Moreover, propagation of osteoblastogenesis inhibition among neighboring osteocytes was reported to be due to secretion of high levels of sclerostin by MM-PCs, a DKK1-dependent factor also known to inhibit the Wnt pathway, leading to decreased bone regeneration (50).

In this context, studies have also shown that MM cells secrete factors as cargo in EVs that have dual functions to both inhibit osteoblast development and stimulate osteoclast differentiation and their bone resorption activity. In a recent study, Zhang et al. found that MM-EVs were enriched in molecules that negatively regulate osteogenesis both in vitro and in vivo and positively correlated with bone lesions in MM patients. By conducting RNA-sequencing, the research group found that a specific regulatory miRNA, miR-103-3p, which plays a crucial role in osteogenesis by targeting the osteoblast developing factor RUNX2, was highly elevated in MM-PC-derived EVs. MMEVs enriched in miR-103-3p were transferred to BM-MSCs, resulting in impairment of osteoblast differentiation (51). Moreover, another study revealed that miR-129 also played a role in vesicle-mediated bone disease. miR-129 was selectively packaged in EVs that were released by MM-PCs but not by PCs of smoldering myeloma, where bone lesions do not occur. MMEVs carrying high levels of miR-129 were selectively taken up by MSCs and inhibited the differentiation of the latter to osteoblasts. Mechanistically, miR-129 inhibited the expression of the transcription factor $\mathrm{Sp} 1$ and its target alkaline phosphatase, both positive modulators of osteoblastic differentiation (52). Other inhibitors of bone growth contributing to osteolysis were also found to be enriched in EVs shed by MM cells and 
transferred to MSCs. Among those factors is DKK-1 mRNA. Once in the recipient cells, DKK-1 mRNA is translated to protein and was shown to downregulate the expression of Runx2, Osterix and Collagen 1A1, all involved in osteoblast differentiation (53).

MM-EVs not only inhibit osteoblast differentiation but also support bone break-down by enhancing osteoclast formation. Raimondi et al. were the first to show that MM-derived EVs induced the migration of pre-osteoclasts and increased their proliferation through activation of the chemokine receptor CXCR4. MM-EVs were also shown to induce the differentiation of pre-osteoclasts to multinuclear mature osteoclasts capable of promoting bone resorption (54). Moreover, MM-PCs cells and MM-BMSCs showed to secrete high levels of osteoclast-activating cytokines such as the receptor activator of nuclear factor $\kappa B$-ligand (RANKL), a key factor and dominant mediator of osteoclast differentiation, activation and survival (55). Additionally, MM-PCs also inhibited the secretion of osteoprotegerin (OG), an important factor involved in osteoblast differentiation. A tight connection between OG and RANKL has been observed. OG acts as a decoy; it antagonizes the biding of RANKL to its receptor, which results in the inhibition of the NK- $\mathrm{BB}$ pathway, thereby preserving the integrity of bone mass. Consequently, a balanced RANKL/OG ratio is essential for maintaining proper bone development (56). In MM, the ratio is significantly disrupted favoring RANKL and NF- $\kappa B$ pathway activation, resulting in the transcription of several downstream factors involved in bone lesions. Pitari et al. detailed the molecular mechanism that causes such a disruption. They showed that both PCs and BMSCs isolated from MM patients secrete high levels of the oncomiRNA miR-21 encapsulated in EVs $(41,57)$. Once transferred to normal BM-MSCs, miR-21 directly targets the 3'UTR of OG, resulting in its degradation and the inhibition of its secretion. Additionally, miR-21 enhances STAT-3 dependent signaling by suppressing PIAS3, the inhibitor of STAT-3, thereby resulting in constitutive STAT3-mediated RANKL gene expression. By both inhibiting OG and activating RANKL, overexpression of miR-21 in the MM microenvironment plays a pivotal role in shifting the balance towards osteoclast proliferation and activation, causing progressive bone resorption (57). Another important osteoclastic factor that is specifically enriched in MM-derived EVs is amphiregulin (AREG). Raimondo et al. reported that EV-AREG shed by MM-PCs were able to induce the differentiation of osteoclasts from their precursors by activating epidermal growth factor receptor and its downstream target, SNAIL. MM-EVs carrying high amounts of AREG not only acted on pre-osteoclasts but were also taken up by human MSCs, leading to increased MM cell adhesion, release of the pro-osteoclastogenic cytokine IL- 8 and inhibition of osteoblast differentiation (58).

In addition to the impaired osteoblast/osteoclast balance that is known to significantly contribute to MM-bone disease, studies have demonstrated the essential role of medullary adipocytes in MM- bone resorption (59). Adipocytes are differentiated from MSCs and secrete metabolically active molecules such as adipokines, growth factors and inflammatory activators, all contributors to loss of bone mass (60). In fact, an increase in both the pre-adipocyte and mature adipocyte numbers as well as adipokine secretion were reported to recruit macrophages into the BM microenvironment, a major source of TNF- $\alpha$. TNF- $\alpha$ induces RANKL activation by adipocytes, which in turn leads to the differentiation of MSCs to osteoclasts (61). Moreover, a crosstalk between adipocytes and osteoblasts was shown to affect the adipocyte/osteoblast balance. For instance, a study revealed that EVs released by adipocytes enriched in adipogenic PPAR $\gamma$, leptin, CEBP $\alpha$ and CEBP $\delta$ mRNAs, as well as the anti-osteoblastic miR138, miR30c, miR125a, miR-125b, and miR-31 -miRNAs were transferred to BM-MSC-derived osteoblasts, resulting in increased expression of adipocytic transcripts, marrow adiposity and subsequent bone lesions (62).

MM-PCs also altered the balance. More recently, Liu et al. demonstrated that a direct contact between MM-PCs and MSCs shifted MSC differentiation toward adipocytes over osteoblasts. The research group showed that Integrin $\alpha 4$ on the surface of MM cells activated the adhesion molecule VCAM1 on MSCs, leading to inhibition of the E3 ubiquitin ligase MURF1, which resulted in stabilization of the adipocyte transcription factor PPAR $\gamma 2$ (63).

In addition to their detrimental role in MM-related bone disease, adipocytes further enhance MM cell growth by releasing secretory factors used as a source of energy by MM-PCs. Hence, several reports demonstrated that elevated levels of adipokines such as leptin, adipsin, and visfatin promoted MM cell proliferation and resistance to chemotherapy (64-66). Moreover, adipocytes induce homing of MM cells to the BM by secreting chemoattractants such as MCP-1 and SDF-1 $\alpha$ (67). Consequently, MM cells in the BM microenvironment adhere to adipocytes that in turn protect MM-PCs from apoptosis-induced chemotherapy (60).

\section{MM-EV-RNAs AND ENDOTHELIAL CELLS}

Endothelial cells are important components of the BM microenvironment. They are part of the vascular niche and play an essential role in the formation of new blood vessels from pre-existing vessels, a physiological process named angiogenesis. In cancer, tumor cell proliferation, survival and dissemination are highly dependent on angiogenesis, as cancerous cells require comparatively more nutrients and oxygen from the vasculature. Blocking endothelial cell proliferation and tube formation is a unique therapeutic approach used to prevent growth and spread of malignant cells (68). In MM, BM angiogenesis is a hallmark of disease progression; it is present in the majority of patients requiring treatment and correlates with poor prognosis (69). A study using primary samples revealed that supernatants of MM-PCs induced significantly higher in vitro angiogenesis compared to that from normal BM-PCs. Most of the secretory molecules that enhanced blood vessel sprouts are known pro-angiogenic factors, such as vascular endothelial growth factor (VEGF), basic fibroblast growth Factor (bFGF), and angiopoietin-1 (70). In fact, EVs secreted by MM cells contributed to angiogenesis by transferring 
high amounts of VEGF to endothelial cells, resulting in their proliferation and capillary structure formation (71). The mechanism underlying the binding of MM-EVs to endothelial cells and the delivery of their cargoes has been recently identified. Purushothaman et al. found that MM-EVs expressing high levels of fibronectin on their surface specifically bind to heparin sulfate on endothelial cells, thus mediating MM-EV-BM endothelial cell interaction (72). Moreover, regulatory RNAs have also been shown to act as key factors in EV-mediated BM angiogenesis. A study reported that MM-EVs carrying high levels of the oncomiRNA miR-135b were selectively transferred to BM endothelial cells. In recipient cells, miR-135b directly suppressed its target, hypoxia-inducer factor 1 (FIH-1), a negative regulator of HIF-1 $\alpha$. As a result, under hypoxic conditions, high levels of pro-angiogenic factors, known to be activated via the HIF1- $\alpha$ signaling pathway, are secreted by endothelial cells, leading to enhanced endothelial tube formation and angiogenesis both in vitro and in vivo. Consequently, EVs released by MM-PCs under hypoxic conditions were shown to carry even higher levels of miR$135 \mathrm{~b}$, creating a positive feedback loop that leads to extremely low oxygen levels in the MM microenvironment to further sustain BM-angiogenesis (73). In addition to microRNAs, piwi-interacting RNAs were shown in a recent report to be important regulators involved in MM angiogenesis. PiRNA823 is encapsulated in EVs, accumulates in the peripheral blood of MM patients and positively correlates with late stage disease and poor prognosis (74). It was also found to mediate intercellular communication between MM-PCs and the BMmicroenvironment. In particular, MM-derived EVs enriched in piRNA-823 were transferred to endothelial cells causing an increase in proliferation, tube formation and invasion by enhancing the expression of VEGF and IL6 and attenuating apoptosis in the target cells (75).

\section{MM-EVs AND IMMUNE CELLS}

Immune cells are important defensive cells located in the BM microenvironment of patients with hematological malignancies. The ability of tumor cells to evade immune surveillance is a key factor for disease progression and metastatic dissemination (76). Tumor derived EVs are believed to participate in the cross-talk between malignant and the surrounding immune cells, resulting in suppression of the immune response in order to create a tumor-supportive environment (77). In MM, the cargoes of tumor derived EVs that play a role in repressing the immune defense are not well characterized. However, studies investigated the biological functions of MM-PC- and MM-BMSC-derived EVs on immune cells of the BM microenvironment. Natural killer (NK) cells, which are an essential component of the innate immune system known to exert cytotoxic effects against microbial infections and tumor cells, are the most among immune cells that are affected by MM-EVs. Xiong et al. first demonstrated that MM-PC-derived EVs significantly reduced the cytotoxic activity of NK cells against MM. The effect was mediated via transferring of repressor factors that induced a dramatic decline in the expression levels of activating receptors NKp46, NKp30 and NKG2D, known to play an essential role in NK cytotoxicity (78). Moreover, another study showed that, under genotoxic stress, MM cells released high amounts of EVs that were selectively taken up by NK cells, resulting in suppression of the latter's activity (79). At the molecular level, transfer of the metalloproteinase ADAM10 was believed to be involved in repression of NK cytotoxicity. ADAM10 is known to shed NKG2D receptor ligand MIC. Once shed, MIC becomes soluble and blocks NKG2D by binding to its activating domain (80). Another type of cargo carried by MM-PC-derived EVs that inhibits NK cell function is the MM-associated antigen CD38. The CD38 molecule is either bound to the surface of PCs or exists in a soluble form that acts as an ectoenzyme that converts nucleotides to adenosine. Adenosine binds to purinergic P2 receptors present on the surface of immune cells, leading to their anergic response. MM-PC-derived EVs were shown to be enriched in the soluble form of CD38 and CD38 mRNA; they were transferred to $\mathrm{NK}$ cells and caused suppression of cytotoxicity against MM cells (81).

Myeloid derived suppressor cells (MDSCs) are another type of immune cell located in the MM-BM microenvironment that showed to play an essential role in MM progression. MDSCs are a small population of immature myeloid cells that suppress the function of effector $\mathrm{T}$ cells, thereby contributing to MM-PC escape from immune surveillance (82). In fact, MM-BMSCs were shown to communicate with MDSCs via EVs. EVs released by BMSCs were transferred to MDSCs and induced the proliferation of the latter in a mechanism dependent on STAT3 and STAT1 signaling pathways. As a result, overgrown MDSCs secreted high levels of nitric oxide in the MM-BM microenvironment, causing $\mathrm{T}$ cell death and escape of MMPCs from cytotoxic T cell attack (83).

Other BM immune cells that are key mediators of MM cell survival are tumor associated macrophages (TAMs). Once differentiated from monocytes, macrophages have the plasticity to polarize to either M1 (tumoricidal) or M2 (tumorigenic) macrophages. M1 macrophages are essential in activating tumor immune response by secreting pro-inflammatory cytokines whereas M2 macrophages produce antiinflammatory cytokines/chemokines that not only suppress the immune response but also contribute to malignant cell proliferation and survival (84). In vivo, M2 macrophages are also named tumor associated macrophages (TAM). In particular, the MM-BM microenvironment was found to be extensively infiltrated with TAMs, and their levels were positively correlated with poor prognosis (85). In addition, TAMs promoted MM progression and metastatic dissemination (86). To our knowledge, we were the first to show the involvement of EVs in a mutual communication between MM-PCs and monocytes/ macrophages, resulting in a shift of the oncogenic landscape of the BM microenvironment. In a recent study, we found that MM-PCs carrying a chromosome 13 aberration (Del13) released abundant amounts of EVs that were deficient in the tumor 
suppressor miR-16. MM-derived EVs were transferred to monocytes, leading to differentiation of the latter to M2 tumor-supportive macrophages. However, EVs released by PCs without Del13 did not affect monocyte differentiation. At the molecular level, we demonstrated that miR-16 targeted the IKK $\alpha / \beta$ complex of the NF- $\kappa B$ canonical pathway, leading to lowered expression of key growth factors and cytokines implicated in M2 differentiation (IL-10, IL-8, TNF- $\alpha$ ). Consequently, in the absence of the transfer of high levels of miR-16 to monocytes, enhanced NF- $\kappa \mathrm{B}$ activity as well as increased expression and production of tolerogenic cytokines/ chemokines essential for M2 macrophage polarization occurs (35).

\section{Role of EV-Non-Coding Regulatory RNAs as Biomarkers in MM}

$\mathrm{MM}$ is a hematological malignancy where diagnosis, prognosis and prediction of disease progression are somewhat challenging because of the lack of powerful monitoring tools for estimating disease outcome and differentiating MM from other neoplasia; therefore, non-invasive biomarkers are urgently need to better monitor the disease and predict treatment response (87). Circulating free regulatory non-coding RNAs (ctrfRNAs) in patients' plasma have proved to be useful predictive tools to monitor disease development. However, the major limitations to use ctrfRNAs as a liquid biopsy is their instability and short half lives in biological fluids (88). EVs encapsulating regulatory RNAs have accordingly been shown to be a comparatively strong noninvasive biomarker platform because of their high abundance in biofluids and their ability to protect their cargoes from nuclease and protease degradation $(30,89)$. In fact, patients with $\mathrm{MM}$ release significantly higher levels of EV-carrying markers such as CD38 and CD138 in their plasma with respect to healthy controls (7). In particular, circulating microRNAs carried by EVs were differentially expressed between MM patients and healthy controls. RNA sequencing on the aforementioned circulating exosomes revealed that, among a large exosomal microRNA panel, only EV-let7b and EV-miR-18a were associated with disease prognosis. Patients with severe disease had low levels of EV-let7b and EV-miR-18a in circulation, and their serum levels negatively correlated with progression-free survival and overall survival (30). Moreover, measuring EVmicroRNA levels is not only a useful tool to monitor MM progression but could also be applicable to discriminate between $\mathrm{MM}$ and its precursor states. For instance, high levels of EV-miR-129 were detected in the BM and sera of patients with MM but not in patients with smoldering myeloma. miR-129 targets several genes involved in osteoblast differentiation, and its overexpression is associated with bone diseases; therefore, circulating EV-miR129 levels could be a suitable indicator of MM bone disease development (52), a complication not observed in MGUS or smoldering myeloma.

We showed that, other than its tumor suppressive role in communicating between MM-PCs cells and cells of the BM microenvironment, EV-miR-16 is among the few microRNAs encapsulated in EVs that were associated with MM prognosis in patients treated with proteasome inhibitor-based therapy. Patients who released high levels of EV-miR-16 in their sera had significantly longer five years overall survival compared to those with lower levels of EV-miR-16 (90). In agreement with these findings, Zhang et al. conducted an exosome-associated miRNA expression pattern on patients resistant to bortezomib. The research group found that exosomal miR-16, miR-15a, miR$20 \mathrm{a}$, and miR-17 were strongly downregulated in circulation and were associated with drug resistance (91). A majority of MM patients develop resistance to bortezomib during the course of treatment; thus, circulating EV-miR-16 and -miR15a could be of important use as biomarkers of treatment response. In addition to microRNAs, lncRNAs are other regulatory RNAs delivered by EVs and are released in large levels in circulation. A recent study showed that the lncRNA LNC00461, which binds miR-15a/16, was highly expressed in MM exosomes (39). Monitoring EVLNC00461 levels in the sera of MM patients could be another approach to predict $\mathrm{MM}$ progression and resistance to bortezomib treatment. Other than their role in $\mathrm{MM}$ prognostication, EVs carrying lncRNAs can also be usedp to distinguish malignancies with monoclonal gammopathies. For instance, the lncRNA content of EVs released in the plasma of MGUS and MM patients were shown to be different. However, among 84 lncRNAs secreted, only one exosomal lncRNA, PRINS (psoriasis susceptibility-related RNA gene induced by stress), was found to be dysregulated in MM and MGUS patients compared to healthy controls. Levels of exosomal PRINS positively correlated with clinical parameters such as bone marrow plasma cell infiltrate rate, albumin, creatinine, and lactate dehydrogenase levels (92). These findings emphasize the potential diagnostic role of circulating EV-PRINS in discriminating monoclonal gammopathies from healthy subjects. The role of MM-EVs as biomarkers of disease and their biological functions are summarized in Table $\mathbf{1}$.

\section{DISCUSSION}

We have shown clear evidence of the involvement of EVs in reeducating the BM microenvironment in favor of MM survival and progression. In particular, we discussed the involvement of ncRNAs contained in EVs derived from MM-PCs in the several cellular compartments of the BM microenvironment that highly impact MM-PC proliferation and escape from immune surveillance, increase angiogenesis and worsen the severity of MM-associated bone disease. Although studies have confirmed the contribution of EV-RNAs in a mutual communication between MM-PCs and different cell types in the BMmicroenvironment, some of which are reviewed above (Figure 1), the mechanisms involved in cell target specificity and route of cellular uptake are yet to be fully identified. For instance, a recent study shed light on the identity of the surface molecules on MM-EVs that played a role in directing EVs towards endothelial cells (72). Similar work should be done to unravel the full $\mathrm{EV}$ receptor molecular spectrum responsible for guiding EVs to specific target cells in the MM-BM microenvironment. 
TABLE 1 | Overview of the role of MM-EV-associated ncRNAs as biomarkers of disease and their biological functions.

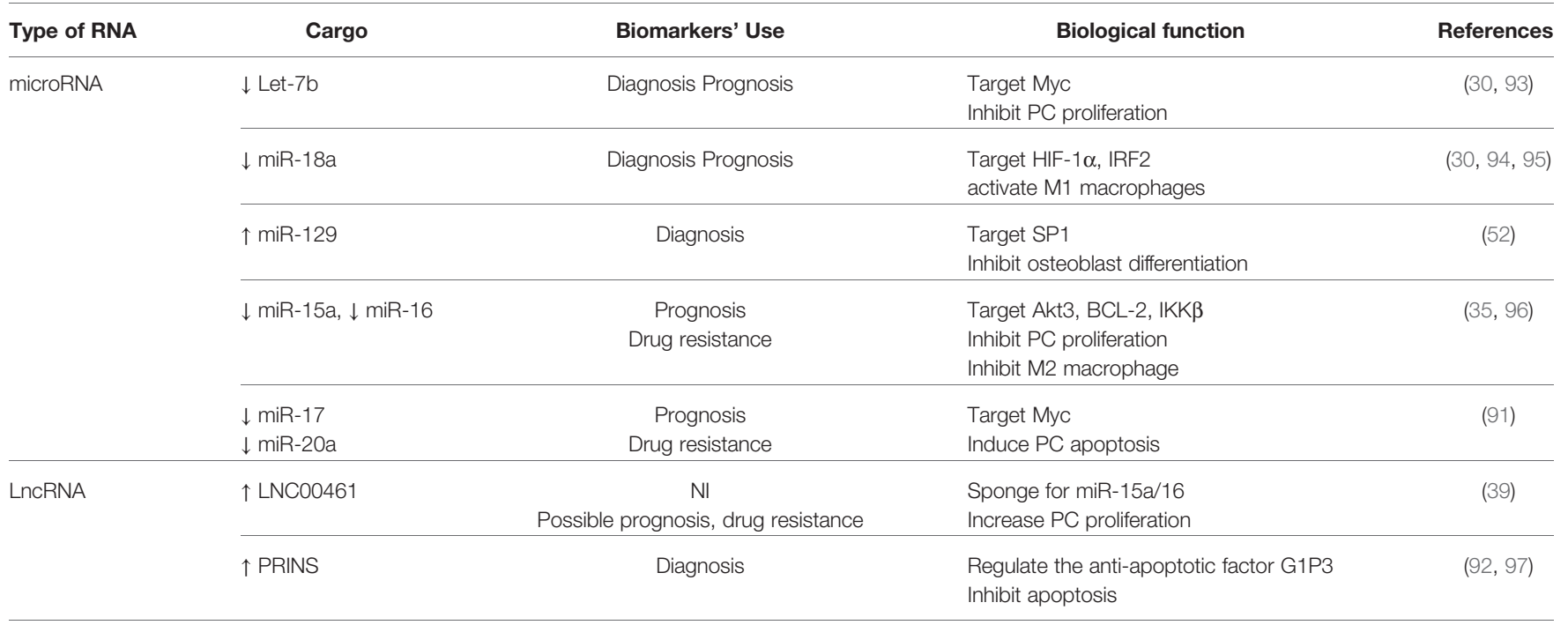

MM, Multiple myeloma; EV, Extracellular vesicle; ncRNA, Non-coding RNA; PC, Plasma cell ; $\downarrow$, Low levels; $\uparrow$, high levels; LncRNA, Long non-coding RNA; NI, Not identified.
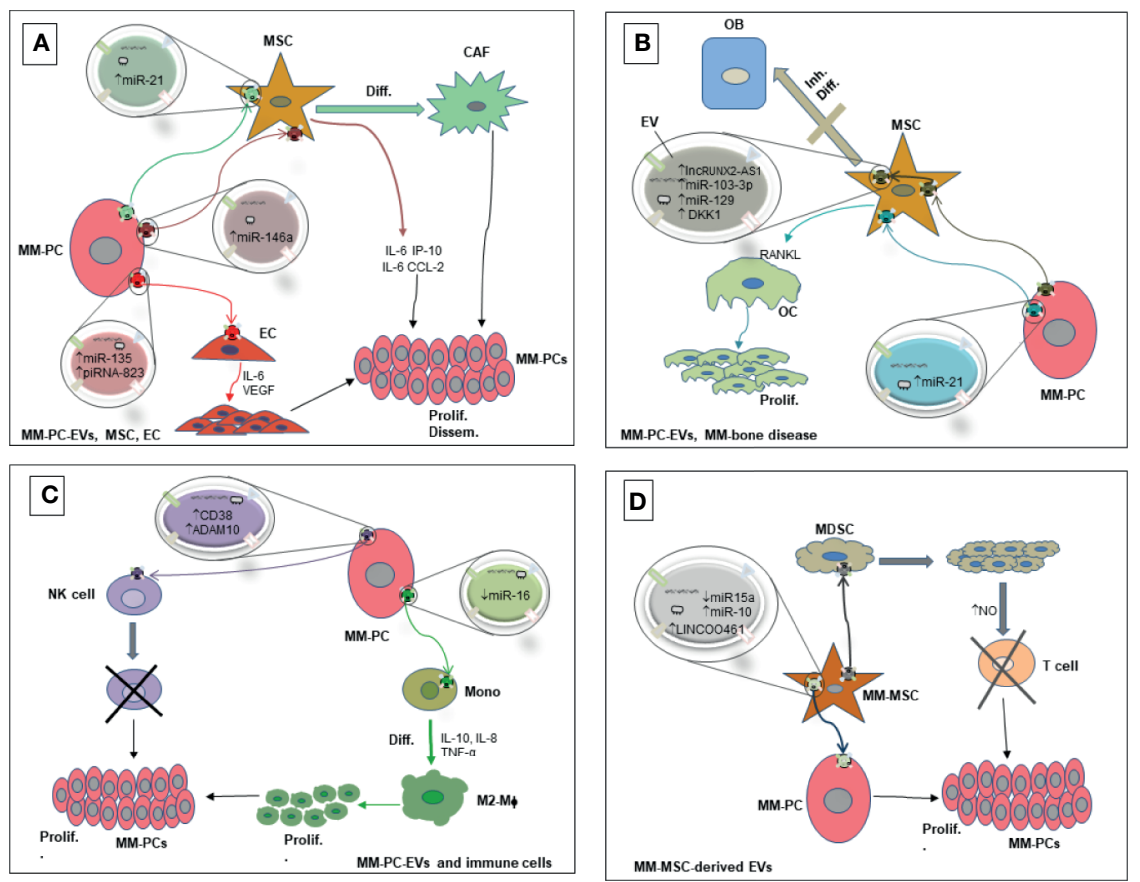

FIGURE 1 | Diagram showing the interaction of MM-PCs with several components of the BM-microenvironment via RNA-containing EVs and their biological effects. (A) Interaction of MM-PC-derived EVs with MSCs and ECs. (B) Interaction of MM-PC-derived EVs with OCs and OBs leading to MM-bone disease. (C) Interaction of MM-PC-derived EVs with immune cells. (D) Interaction of MM-MSC-derived EVs with MM-PCs and other cellular components of the MM microenvironment. EV, Extracellular Vesicle; MM-PC, Multiple myeloma plasma cell; MDSC, Myeloid derived suppressor cell; MM-MSC, Multiple myeloma derived mesenchymal stromal cell; MSC, mesenchymal stromal cell; CAF, Cancer associated fibroblast; OB, Osteoblast; OC, Osteoclast; EC, Endothelial cell; Mono, Monocyte; M2-M申, M2 macrophage; NK cell, Natural killer cell; RANKL, receptor activator of nuclear factor kB-ligand; NO, Nitric oxide; Diff., Differentiation; Inh. Diff., Inhibition of differentiation; prolif., Proliferation; Dissem., Dissemination; $\uparrow$, High levels; $\downarrow$, Low levels.

Moreover, MM-EV-associated ncRNAs are highly abundant in circulation, and their signature is different from those released by non-cancerous cells (7); therefore, as their analysis is novel, they are being considered as distinct and feasible non-invasive biomarkers of disease occurrence, progression and resistance to therapy. Because EV contents reflect the cells where they originated from (18), other than being useful prognostic and diagnostic tools, EVs containing RNAs released by MM cells or 
cells of the microenvironment are considered tumor specific EVs, rendering them potential therapeutic targets. In this context, suppression of EV biogenesis or secretion could be a plausible therapeutic approach in MM. However, it could result in both favorable and unfavorable consequences because of the essential role in maintaining normal physiological responses by EVs that are released by healthy cells (98). Hence, applying innovative technologies that simultaneously characterize and specifically detect tumor-associated EVs on the basis of molecular identity

\section{REFERENCES}

1. Plasma Cell Neoplasms (Including Multiple Myeloma) Treatment ( $\mathrm{PDQ}^{\circledR}{ }^{\circledR}$ Health Professional Version. National Cancer Institute. 11 February 2021. Available at: https://www.cancer.gov/types/myeloma/hp/myelomatreatment-pdq (Accessed on 17 March 2021).

2. Manier S, Sacco A, Leleu X, Ghobrial IM, Roccaro AM. Bone Marrow Microenvironment in Multiple Myeloma Progression. J Biomed Biotechnol (2012) 2012:157496. doi: 10.1155/2012/157496

3. Maas SLN, Breakefield XO, Weaver AM. Extracellular Vesicles: Unique Intercellular Delivery Vehicles. Trends Cell Biol (2017) 27(3):172-88. doi: $10.1016 /$ j.tcb.2016.11.003

4. Wolf P. The Nature and Significance of Platelet Products in Human Plasma. Br J Haematol (1967) 13(3):269-88. doi: 10.1111/j.1365-2141.1967. tb08741.x

5. Muralidharan-Chari V, Clancy JW, Sedgwick A, D'Souza-Schorey C. Microvesicles: Mediators of Extracellular Communication During Cancer Progression. J Cell Sci (2010) 123(Pt 10):1603-11. doi: 10.1242/jcs.064386

6. Colombo M, Giannandrea D, Lesma E, Basile A, Chiaramonte R. Extracellular Vesicles Enhance Multiple Myeloma Metastatic Dissemination. Int J Mol Sci (2019) 20(13)3236-51. doi: 10.3390/ijms20133236

7. Caivano A, Laurenzana I, De Luca L, La Rocca F, Simeon V, Trino S, et al. High Serum Levels of Extracellular Vesicles Expressing Malignancy-Related Markers Are Released in Patients With Various Types of Hematological Neoplastic Disorders. Tumour Biol J Int Soc Oncodev Biol Med (2015) 36 (12):9739-52. doi: 10.1007/s13277-015-3741-3

8. Li M, Zeringer E, Barta T, Schageman J, Cheng A, Vlassov AV. Analysis of the RNA Content of the Exosomes Derived From Blood Serum and Urine and Its Potential as Biomarkers. Philos Trans R Soc London Ser B Biol Sci (2014) 369 (1652):20130502. doi: 10.1098/rstb.2013.0502

9. Lässer C, Shelke GV, Yeri A, Kim DK, Crescitelli R, Raimondo S, et al. Two Distinct Extracellular RNA Signatures Released by a Single Cell Type Identified by Microarray and Next-Generation Sequencing. RNA Biol (2017) 14(1):58-72. doi: 10.1080/15476286.2016.1249092

10. Yuan T, Huang X, Woodcock M, Du M, Dittmar R, Wang Y, et al. Plasma Extracellular RNA Profiles in Healthy and Cancer Patients. Sci Rep (2016) 6:19413. doi: 10.1038/srep19413

11. Escudier B, Dorval T, Chaput N, André F, Caby MP, Novault S, et al. Vaccination of Metastatic Melanoma Patients With Autologous Dendritic Cell (DC) Derived-Exosomes: Results of The First Phase I Clinical Trial. J Trans Med (2005) 3(1):10. doi: 10.1186/1479-5876-3-10

12. Morse MA, Garst J, Osada T, Khan S, Hobeika A, Clay TM, et al. A Phase I Study of Dexosome Immunotherapy in Patients With Advanced NonSmall Cell Lung Cancer. J Trans Med (2005) 3(1):9. doi: 10.1186/14795876-3-9

13. Dai S, Wei D, Wu Z, Zhou X, Wei X, Huang H, et al. Phase I Clinical Trial of Autologous Ascites-Derived Exosomes Combined With GM-CSF for Colorectal Cancer. Mol Ther J Am Soc Gene Ther (2008) 16(4):782-90. doi: $10.1038 / \mathrm{mt} .2008 .1$

14. Théry C, Witwer KW, Aikawa E, Alcaraz MJ, Anderson JD, Andriantsitohaina R, et al. Minimal Information for Studies of Extracellular Vesicles 2018 (MISEV2018): A Position Statement of the International Society for Extracellular Vesicles and Update of the MISEV2014 Guidelines. J Extracell Vesicles (2018) 7(1):1535750. doi: 10.1080/20013078.2018.1535750 of their cargoes is needed. Thanks to modern high-throughput technology and development of multi-omics analysis techniques, targeting tumor specific EVs in MM could become possible.

\section{AUTHOR CONTRIBUTIONS}

JK, JS, and FP wrote the manuscript. All authors contributed to the article and approved the submitted version.

15. Kowal J, Arras G, Colombo M, Jouve M, Morath JP, Primdal-Bengtson B, et al. Proteomic Comparison Defines Novel Markers to Characterize Heterogeneous Populations of Extracellular Vesicle Subtypes. Proc Natl Acad Sci USA (2016) 113(8):E968-77. doi: 10.1073/pnas.1521230113

16. Willms E, Johansson HJ, Mäger I, Lee Y, Blomberg KE, Sadik M, et al. Cells Release Subpopulations of Exosomes With Distinct Molecular and Biological Properties. Sci Rep (2016) 6:22519. doi: 10.1038/srep22519

17. Avci E. Balci-Peynircioglu. An Overview of Exosomes: From Biology to Emerging Roles in Immune Response. Acta Med (2015) 47(1):2-10.

18. Raposo G, Stoorvogel W. Extracellular Vesicles: Exosomes, Microvesicles, and Friends. J Cell Biol (2013) 200(4):373-83. doi: 10.1083/jcb.201211138

19. Timár CI, Lorincz AM, Csépányi-Kömi R, Vályi-Nagy A, Nagy G, Buzás EI, et al. Antibacterial Effect of Microvesicles Released from Human Neutrophilic Granulocytes. Blood (2013) 121(3):510-8. doi: 10.1182/ blood-2012-05-431114

20. Johnson SM, Dempsey C, Parker C, Mironov A, Bradley H, Saha V. Acute Lymphoblastic Leukaemia Cells Produce Large Extracellular Vesicles Containing Organelles and an Active Cytoskeleton. J Extracell Vesicles (2017) 6(1):1294339. doi: 10.1080/20013078.2017.1294339

21. Di Vizio D, Morello M, Dudley AC, Schow PW, Adam RM, Morley S, et al. Large Oncosomes in Human Prostate Cancer Tissues and in the Circulation of Mice With Metastatic Disease. Am J Pathol (2012) 181(5):1573-84. doi: 10.1016/j.ajpath.2012.07.030

22. Taylor RC, Cullen SP, Martin SJ. Apoptosis: Controlled Demolition at the Cellular Level. Nat Rev Mol Cell Biol (2008) 9(3):231-41. doi: 10.1038/ nrm 2312

23. Ciardiello C, Cavallini L, Spinelli C, Yang J, Reis-Sobreiro M, de Candia P, et al. Focus on Extracellular Vesicles: New Frontiers of Cell-to-Cell Communication in Cancer. Int J Mol Sci (2016) 17(2):175. doi: 10.3390/ ijms 17020175

24. Khalife J, Sanchez JF, Pichiorri F. Extracellular Vesicles in Hematological Malignancies: From Biomarkers to Therapeutic Tools. Diagn (Basel Switzerland) (2020) 10(12):1065. doi: 10.3390/diagnostics10121065

25. Hong CS, Muller L, Whiteside TL, Boyiadzis M. Plasma Exosomes as Markers of Therapeutic Response in Patients With Acute Myeloid Leukemia. Front Immunol (2014) 5:160. doi: 10.3389/fimmu.2014.00160

26. De Luca L, Laurenzana I, Trino S, Lamorte D, Caivano A, Musto P. An Update on Extracellular Vesicles in Multiple Myeloma: A Focus on Their Role in Cellto-Cell Cross-Talk and as Potential Liquid Biopsy Biomarkers. Expert Rev Mol Diagn (2019) 19(3):249-58. doi: 10.1080/14737159.2019.1583103

27. Muller L, Hong CS, Stolz DB, Watkins SC, Whiteside TL. Isolation of Biologically-Active Exosomes from Human Plasma. I Immunol Methods (2014) 411:55-65. doi: 10.1016/j.jim.2014.06.007

28. Tanasi I, Adamo A, Kamga PT, Bazzoni R, Krampera M. High-Throughput Analysis and Functional Interpretation of Extracellular Vesicle Content in Hematological Malignancies. Comput Struct Biotechnol J (2020) 18:2670-7. doi: 10.1016/j.csbj.2020.09.027

29. Harshman SW, Canella A, Ciarlariello PD, Agarwal K, Branson OE, Rocci A, et al. Proteomic Characterization of Circulating Extracellular Vesicles Identifies Novel Serum Myeloma Associated Markers. J Proteomics (2016) 136:89-98. doi: 10.1016/j.jprot.2015.12.016

30. Manier S, Liu CJ, Avet-Loiseau H, Park J, Shi J, Campigotto F, et al. Prognostic Role of Circulating Exosomal miRNAs in Multiple Myeloma. Blood (2017) 129(17):2429-36. doi: 10.1182/blood-2016-09-742296 
31. Seckinger A, Meißner T, Moreaux J, Benes V, Hillengass J, Castoldi M, et al. miRNAs in Multiple Myeloma-A Survival Relevant Complex Regulator of Gene Expression. Oncotarget (2015) 6(36):39165-83. doi: 10.18632/ oncotarget.5381

32. Chen BZ, Morgan GJ, Barlogie B, Epstein J. Specific Exosomal Microrna Are Differentially Expressed Between High and Low-Risk Myeloma Suggesting They Are Pathogenically Important. Blood (2015) 126(23):4189.

33. Roccaro AM, Sacco A, Maiso P, Azab AK, Tai YT, Reagan M, et al. BM Mesenchymal Stromal Cell-Derived Exosomes Facilitate Multiple Myeloma Progression. J Clin Invest (2013) 123(4):1542-55. doi: 10.1172/jci66517

34. Umezu T, Imanishi S, Yoshizawa S, Kawana C, Ohyashiki JH, Ohyashiki K. Induction of Multiple Myeloma Bone Marrow Stromal Cell Apoptosis by Inhibiting Extracellular Vesicle miR-10a Secretion. Blood Adv (2019) 3 (21):3228-40. doi: 10.1182/bloodadvances.2019000403

35. Khalife J, Ghose J, Martella M, Viola D, Rocci A, Troadec E, et al. MiR-16 Regulates Crosstalk in NF- $\kappa b$ Tolerogenic Inflammatory Signaling Between Myeloma Cells and Bone Marrow Macrophages. JCI Insight (2019) 4(21). doi: 10.1172 /jci.insight.129348

36. Papaccio F, Paino F, Regad T, Papaccio G, Desiderio V, Tirino V. Concise Review: Cancer Cells, Cancer Stem Cells, and Mesenchymal Stem Cells: Influence in Cancer Development. Stem Cells Trans Med (2017) 6 (12):2115-25. doi: 10.1002/sctm.17-0138

37. Ribatti D, Moschetta M, Vacca A. Microenvironment and Multiple Myeloma Spread. Thromb Res (2014) 133(Suppl 2):S102-6. doi: 10.1016/s0049-3848(14) 50017-5

38. Wang J, Hendrix A, Hernot S, Lemaire M, De Bruyne E, Van Valckenborgh E, et al. Bone Marrow Stromal Cell-Derived Exosomes as Communicators in Drug Resistance in Multiple Myeloma Cells. Blood (2014) 124(4):555-66. doi: 10.1182/blood-2014-03-562439

39. Deng M, Yuan H, Liu S, Hu Z, Xiao H. Exosome-Transmitted LINC00461 Promotes Multiple Myeloma Cell Proliferation and Suppresses Apoptosis by Modulating microRNA/BCL-2 Expression. Cytotherapy (2019) 21(1):96-106. doi: 10.1016/j.jcyt.2018.10.006

40. Yaccoby S, Wezeman MJ, Henderson A, Cottler-Fox M, Yi Q, Barlogie B, et al. Cancer and the Microenvironment: Myeloma-Osteoclast Interactions as a Model. Cancer Res (2004) 64(6):2016-23. doi: 10.1158/0008-5472.can-03-1131

41. Cheng Q, Li X, Liu J, Ye Q, Chen Y, Tan S, et al. Multiple Myeloma-Derived Exosomes Regulate the Functions of Mesenchymal Stem Cells Partially Via Modulating miR-21 and Mir-146a. Stem Cells Int (2017) 2017:9012152. doi: $10.1155 / 2017 / 9012152$

42. De Veirman K, Wang J, Xu S, Leleu X, Himpe E, Maes K, et al. Induction of miR-146a by Multiple Myeloma Cells in Mesenchymal Stromal Cells Stimulates Their Pro-Tumoral Activity. Cancer Lett (2016) 377(1):17-24. doi: 10.1016/j.canlet.2016.04.024

43. Li B, Xu H, Han H, Song S, Zhang X, Ouyang L, et al. Exosome-Mediated Transfer of lncRUNX2-AS1 From Multiple Myeloma Cells to MSCs Contributes to Osteogenesis. Oncogene (2018) 37(41):5508-19. doi: 10.1038/ s41388-018-0359-0

44. Hameed A, Brady JJ, Dowling P, Clynes M, O'Gorman P. Bone Disease in Multiple Myeloma: Pathophysiology and Management. Cancer Growth Metastasis (2014) 7:33-42. doi: 10.4137/cgm.S16817

45. Blair HC, Zaidi M, Huang CL, Sun L. The Developmental Basis of Skeletal Cell Differentiation and the Molecular Basis of Major Skeletal Defects. Biol Rev Camb Philos Soc (2008) 83(4):401-15. doi: 10.1111/j.1469-185X.2008.00048.x

46. Sezer O, Heider U, Jakob C, Zavrski I, Eucker J, Possinger K, et al. Immunocytochemistry Reveals RANKL Expression of Myeloma Cells. Blood (2002) 99(12):4646-7; author reply 7. doi: 10.1182/blood-2002-01-0148

47. Fu J, Li S, Feng R, Ma H, Sabeh F, Roodman GD, et al. Multiple MyelomaDerived MMP-13 Mediates Osteoclast Fusogenesis and Osteolytic Disease. J Clin Invest (2016) 126(5):1759-72. doi: 10.1172/jci80276

48. Colucci S, Brunetti G, Mori G, Oranger A, Centonze M, Mori C, et al. Soluble Decoy Receptor 3 Modulates the Survival and Formation of Osteoclasts From Multiple Myeloma Bone Disease Patients. Leukemia (2009) 23(11):2139-46. doi: 10.1038/leu.2009.136

49. Qiang YW, Barlogie B, Rudikoff S, Shaughnessy JD Jr. Dkk1-induced Inhibition of Wnt Signaling in Osteoblast Differentiation Is An Underlying Mechanism of Bone Loss in Multiple Myeloma. Bone (2008) 42(4):669-80. doi: 10.1016/j.bone.2007.12.006
50. Eda H, Santo L, Wein MN, Hu DZ, Cirstea DD, Nemani N, et al. Regulation of Sclerostin Expression in Multiple Myeloma by Dkk-1: A Potential Therapeutic Strategy for Myeloma Bone Disease. J Bone Miner Res Off J Am Soc Bone Miner Res (2016) 31(6):1225-34. doi: 10.1002/jbmr.2789

51. Zhang L, Lei Q, Wang H, Xu C, Liu T, Kong F, et al. Tumor-Derived Extracellular Vesicles Inhibit Osteogenesis and Exacerbate Myeloma Bone Disease. Theranostics (2019) 9(1):196-209. doi: 10.7150/thno.27550

52. Raimondo S, Urzì O, Conigliaro A, Bosco GL, Parisi S, Carlisi M, et al. Extracellular Vesicle Micrornas Contribute to the Osteogenic Inhibition of Mesenchymal Stem Cells in Multiple Myeloma. Cancers (2020) 12(2):449. doi: 10.3390/cancers12020449

53. Faict S, Muller J, De Veirman K, De Bruyne E, Maes K, Vrancken L, et al. Exosomes Play a Role in Multiple Myeloma Bone Disease and Tumor Development by Targeting Osteoclasts and Osteoblasts. Blood Cancer J (2018) 8(11):105. doi: 10.1038/s41408-018-0139-7

54. Raimondi L, De Luca A, Amodio N, Manno M, Raccosta S, Taverna S, et al. Involvement of Multiple Myeloma Cell-Derived Exosomes in Osteoclast Differentiation. Oncotarget (2015) 6(15):13772-89. doi: 10.18632/oncotarget.3830

55. Anderson KC, Carrasco RD. Pathogenesis of Myeloma. Annu Rev Pathol (2011) 6:249-74. doi: 10.1146/annurev-pathol-011110-130249

56. Giuliani N, Bataille R, Mancini C, Lazzaretti M, Barillé S. Myeloma Cells Induce Imbalance in the Osteoprotegerin/Osteoprotegerin Ligand System in the Human Bone Marrow Environment. Blood (2001) 98(13):352733.doi: 10.1182/blood.v98.13.3527

57. Pitari MR, Rossi M, Amodio N, Botta C, Morelli E, Federico C, et al. Inhibition of miR-21 Restores RANKL/OPG Ratio in Multiple MyelomaDerived Bone Marrow Stromal Cells and Impairs the Resorbing Activity of Mature Osteoclasts. Oncotarget (2015) 6(29):27343-58. doi: 10.18632/ oncotarget. 4398

58. Raimondo S, Saieva L, Vicario E, Pucci M, Toscani D, Manno M, et al. Multiple Myeloma-Derived Exosomes are Enriched of Amphiregulin (AREG) and Activate the Epidermal Growth Factor Pathway in the Bone Microenvironment Leading to Osteoclastogenesis. J Hematol Oncol (2019) 12(1):2. doi: 10.1186/s13045-018-0689-y

59. Duque G. Bone and Fat Connection in Aging Bone. Curr Opin Rheumatol (2008) 20(4):429-34. doi: 10.1097/BOR.0b013e3283025e9c

60. Caers J, Deleu S, Belaid Z, De Raeve H, Van Valckenborgh E, De Bruyne E, et al. Neighboring Adipocytes Participate in the Bone Marrow Microenvironment of Multiple Myeloma Cells. Leukemia (2007) 21 (7):1580-4. doi: 10.1038/sj.leu.2404658

61. Goto H, Hozumi A, Osaki M, Fukushima T, Sakamoto K, Yonekura A, et al. Primary Human Bone Marrow Adipocytes Support TNF- $\alpha$-Induced Osteoclast Differentiation and Function Through RANKL Expression. Cytokine (2011) 56(3):662-8. doi: 10.1016/j.cyto.2011.09.005

62. Martin PJ, Haren N, Ghali O, Clabaut A, Chauveau C, Hardouin P, et al. Adipogenic RNAs Are Transferred in Osteoblasts Via Bone Marrow Adipocytes-Derived Extracellular Vesicles (Evs). BMC Cell Biol (2015) 16:10. doi: 10.1186/s12860-015-0057-5

63. Liu Z, Liu H, He J, Lin P, Tong Q, Yang J. Myeloma Cells Shift Osteoblastogenesis to Adipogenesis by Inhibiting the Ubiquitin Ligase MURF1 in Mesenchymal Stem Cells. Sci Signaling (2020) 13(633):eaay8203. doi: $10.1126 /$ scisignal.aay 8203

64. Reseland JE, Reppe S, Olstad OK, Hjorth-Hansen H, Brenne AT, Syversen U, et al. Abnormal Adipokine Levels and Leptin-Induced Changes in Gene Expression Profiles in Multiple Myeloma. Eur J Haematol (2009) 83(5):46070. doi: 10.1111/j.1600-0609.2009.01311.x

65. Liu Z, Xu J, He J, Liu H, Lin P, Wan X, et al. Mature Adipocytes in Bone Marrow Protect Myeloma Cells Against Chemotherapy Through Autophagy Activation. Oncotarget (2015) 6(33):34329-41. doi: 10.18632/oncotarget.6020

66. Dalamaga M, Karmaniolas K, Panagiotou A, Hsi A, Chamberland J, Dimas C, et al. Low Circulating Adiponectin and Resistin, But Not Leptin, Levels Are Associated With Multiple Myeloma Risk: A Case-Control Study. Cancer Causes Control CCC (2009) 20(2):193-9. doi: 10.1007/s10552-008-9233-7

67. Trotter TN, Gibson JT, Sherpa TL, Gowda PS, Peker D, Yang Y. AdipocyteLineage Cells Support Growth and Dissemination of Multiple Myeloma in Bone. Am J Pathol (2016) 186(11):3054-63. doi: 10.1016/j.ajpath.2016.07.012

68. Kerbel RS. Tumor Angiogenesis: Past, Present and the Near Future. Carcinogenesis (2000) 21(3):505-15. doi: 10.1093/carcin/21.3.505 
69. Vacca A, Ribatti D, Roncali L, Ranieri G, Serio G, Silvestris F, et al. Bone Marrow Angiogenesis and Progression in Multiple Myeloma. Br J Haematol (1994) 87(3):503-8. doi: 10.1111/j.1365-2141.1994.tb08304.x

70. Hose D, Moreaux J, Meissner T, Seckinger A, Goldschmidt H, Benner A, et al. Induction of Angiogenesis by Normal and Malignant Plasma Cells. Blood (2009) 114(1):128-43. doi: 10.1182/blood-2008-10-184226

71. Liu Y, Zhu XJ, Zeng C, Wu PH, Wang HX, Chen ZC, et al. Microvesicles Secreted From Human Multiple Myeloma Cells Promote Angiogenesis. Acta Pharmacol Sin (2014) 35(2):230-8. doi: 10.1038/aps.2013.141

72. Purushothaman A, Bandari SK, Liu J, Mobley JA, Brown EE, Sanderson RD. Fibronectin on the Surface of Myeloma Cell-Derived Exosomes Mediates Exosome-Cell Interactions. J Biol Chem (2016) 291(4):1652-63. doi: 10.1074/ jbc.M115.686295

73. Umezu T, Tadokoro H, Azuma K, Yoshizawa S, Ohyashiki K, Ohyashiki JH. Exosomal miR-135b Shed From Hypoxic Multiple Myeloma Cells Enhances Angiogenesis by Targeting Factor-Inhibiting HIF-1. Blood (2014) 124 (25):3748-57. doi: 10.1182/blood-2014-05-576116

74. Yan H, Wu QL, Sun CY, Ai LS, Deng J, Zhang L, et al. piRNA-823 Contributes to Tumorigenesis by Regulating De Novo DNA Methylation and Angiogenesis in Multiple Myeloma. Leukemia (2015) 29(1):196-206. doi: $10.1038 /$ leu.2014.135

75. Li B, Hong J, Hong M, Wang Y, Yu T, Zang S, et al. piRNA-823 Delivered by Multiple Myeloma-Derived Extracellular Vesicles Promoted Tumorigenesis Through Re-Educating Endothelial Cells in the Tumor Environment. Oncogene (2019) 38(26):5227-38. doi: 10.1038/s41388-019-0788-4

76. Hanahan D, Weinberg RA. Hallmarks of Cancer: The Next Generation. Cell (2011) 144(5):646-74. doi: 10.1016/j.cell.2011.02.013

77. Lucchetti D, Ricciardi Tenore C, Colella F, Sgambato A. Extracellular Vesicles and Cancer: A Focus on Metabolism, Cytokines, and Immunity. Cancers (2020) 12(1):171. doi: 10.3390/cancers 12010171

78. Xiong WJ, Liu HX, Shi DY, Lou J, Zhang QL. Effect of Myeloma-Derived Exosomes on Surface Activating Receptors of NK Cells. Zhongguo shi yan xue ye xue za zhi (2017) 25(6):1713-7. doi: 10.7534/j.issn.10092137.2017.06.024

79. Vulpis E, Cecere F, Molfetta R, Soriani A, Fionda C, Peruzzi G, et al. Genotoxic Stress Modulates the Release of Exosomes From Multiple Myeloma Cells Capable of Activating NK Cell Cytokine Production: Role of HSP70/TLR2/NF-kB Axis. Oncoimmunology (2017) 6(3):e1279372. doi: 10.1080/2162402x.2017.1279372

80. Zingoni A, Cecere F, Vulpis E, Fionda C, Molfetta R, Soriani A, et al. Genotoxic Stress Induces Senescence-Associated Adam10-Dependent Release of NKG2D Mic Ligands in Multiple Myeloma Cells. J Immunol (Baltimore Md 1950) (2015) 195(2):736-48. doi: 10.4049/jimmunol.1402643

81. Chillemi A, Quarona V, Antonioli L, Ferrari D, Horenstein AL, Malavasi F. Roles and Modalities of Ectonucleotidases in Remodeling the Multiple Myeloma Niche. Front Immunol (2017) 8:305. doi: 10.3389/fimmu.2017. 00305

82. Malek E, de Lima M, Letterio JJ, Kim BG, Finke JH, Driscoll JJ, et al. MyeloidDerived Suppressor Cells: The Green Light for Myeloma Immune Escape. Blood Rev (2016) 30(5):341-8. doi: 10.1016/j.blre.2016.04.002

83. Wang J, De Veirman K, De Beule N, Maes K, De Bruyne E, Van Valckenborgh E, et al. The Bone Marrow Microenvironment Enhances Multiple Myeloma Progression by Exosome-Mediated Activation of Myeloid-Derived Suppressor Cells. Oncotarget (2015) 6(41):43992-4004. doi: 10.18632/oncotarget.6083

84. Shapouri-Moghaddam A, Mohammadian S, Vazini H, Taghadosi M, Esmaeili SA, Mardani F, et al. Macrophage Plasticity, Polarization, and Function in Health and Disease. J Cell Physiol (2018) 233(9):6425-40. doi: $10.1002 /$ jcp. 26429

85. Wang H, Hu WM, Xia ZJ, Liang Y, Lu Y, Lin SX, et al. High Numbers of CD163+ Tumor-Associated Macrophages Correlate With Poor Prognosis in
Multiple Myeloma Patients Receiving Bortezomib-Based Regimens. J Cancer (2019) 10(14):3239-45. doi: 10.7150/jca.30102

86. Zeissig MN, Zannettino ACW, Vandyke K. Tumour Dissemination in Multiple Myeloma Disease Progression and Relapse: A Potential Therapeutic Target in High-Risk Myeloma. Cancers (2020) 12(12):3643. doi: $10.3390 /$ cancers 12123643

87. Iaccino E, Mimmi S, Dattilo V, Marino F, Candeloro P, Di Loria A, et al. Monitoring Multiple Myeloma by Idiotype-Specific Peptide Binders of Tumor-Derived Exosomes. Mol Cancer (2017) 16(1):159. doi: 10.1186/ s12943-017-0730-8

88. Ilie M, Hofman V, Long E, Bordone O, Selva E, Washetine K, et al. Current Challenges for Detection of Circulating Tumor Cells and Cell-Free Circulating Nucleic Acids, and Their Characterization in Non-Small Cell Lung Carcinoma Patients. What is the Best Blood Substrate for Personalized Medicine? Ann Trans Med (2014) 2(11):107. doi: 10.3978/j.issn.23055839.2014.08.11

89. Mader S, Pantel K. Liquid Biopsy: Current Status and Future Perspectives. Oncol Res Treat (2017) 40(7-8):404-8. doi: 10.1159/000478018

90. Rocci A, Hofmeister CC, Geyer S, Stiff A, Gambella M, Cascione L, et al. Circulating miRNA Markers Show Promise as New Prognosticators for Multiple Myeloma. Leukemia (2014) 28(9):1922-6. doi: 10.1038/leu.2014.155

91. Zhang L, Pan L, Xiang B, Zhu H, Wu Y, Chen M, et al. Potential Role of ExosomeAssociated microRNA Panels and In Vivo Environment to Predict Drug Resistance for Patients With Multiple Myeloma. Oncotarget (2016) 7(21):30876-91. doi: 10.18632/oncotarget.9021

92. Sedlarikova L, Bollova B, Radova L, Brozova L, Jarkovsky J, Almasi M, et al. Circulating Exosomal Long Noncoding RNA Prins-First Findings in Monoclonal Gammopathies. Hematol Oncol (2018) 36(5):786-91. doi: 10.1002/hon.2554

93. Manier S, Powers JT, Sacco A, Glavey SV, Huynh D, Reagan MR, et al. The LIN28B/let-7 Axis Is a Novel Therapeutic Pathway in Multiple Myeloma. Leukemia (2017) 31(4):853-60. doi: 10.1038/leu.2016.296

94. Krutilina R, Sun W, Sethuraman A, Brown M, Seagroves TN, Pfeffer LM, et al. MicroRNA-18a Inhibits Hypoxia-Inducible Factor $1 \alpha$ Activity and Lung Metastasis in Basal Breast Cancers. Breast Cancer Res BCR (2014) 16(4): R78. doi: $10.1186 /$ bcr3693

95. Teng Y, Mu J, Hu X, Samykutty A, Zhuang X, Deng Z, et al. GrapefruitDerived Nanovectors Deliver miR-18a for Treatment of Liver Metastasis of Colon Cancer by Induction of M1 Macrophages. Oncotarget (2016) 7 (18):25683-97. doi: 10.18632/oncotarget.8361

96. Roccaro AM, Sacco A, Thompson B, Leleu X, Azab AK, Azab F, et al. MicroRNAs 15a and 16 Regulate Tumor Proliferation in Multiple Myeloma. Blood (2009) 113(26):6669-80. doi: 10.1182/blood-2009-01-198408

97. Szegedi K, Sonkoly E, Nagy N, Németh IB, Bata-Csörgo Z, Kemény L, et al. The Anti-Apoptotic Protein G1P3 Is Overexpressed in Psoriasis and Regulated by the non-Coding RNA, Prins. Exp Dermatol (2010) 19(3):26978. doi: 10.1111/j.1600-0625.2010.01066.x

98. Whiteside TL. Tumor-Derived Exosomes and Their Role in Cancer Progression. Adv Clin Chem (2016) 74:103-41. doi: 10.1016/bs.acc.2015.12.005

Conflict of Interest: The authors declare that the research was conducted in the absence of any commercial or financial relationships that could be construed as a potential conflict of interest.

Copyright (c) 2021 Khalife, Sanchez and Pichiorri. This is an open-access article distributed under the terms of the Creative Commons Attribution License (CC BY). The use, distribution or reproduction in other forums is permitted, provided the original author(s) and the copyright owner(s) are credited and that the original publication in this journal is cited, in accordance with accepted academic practice. No use, distribution or reproduction is permitted which does not comply with these terms. 